\title{
Short Communication: Landmark-based geometric morphometric analysis of wings to distinguish the sex of Aedes mosquito vectors in Thailand
}

\author{
TANAWAT CHAIPHONGPACHARA ${ }^{1, \boldsymbol{\nu}}$, SEDTHAPONG LAOJUN ${ }^{2}$ \\ ${ }^{1}$ College of Allied Health Science, Suan Sunandha Rajabhat University. Samut Songkhram 75000, Thailand \\ Tel./fax. +66-835-865775, `email: tanawat.ch@ssru.ac.th \\ ${ }^{2}$ Program of Public Health, College of Allied Health Sciences, Suan Sunandha Rajabhat University. Samut Songkhram 75000, Thailand
}

Manuscript received: 26 December 2018. Revision accepted: 22 January 2019.

\begin{abstract}
Chaiphongpachara T, Laojun S. 2019. Short Communication: Landmark-based geometric morphometric analysis of wings to distinguish the sex of Aedes mosquito vectors in Thailand. Biodiversitas 20: 419-424. Aedes mosquitoes (Diptera: Culicidae) are medically important insects which are vectors of yellow fever, dengue fever, chikungunya, West Nile, and the Zika virus, emerging problems worldwide. Typically, male )non-vector( and female )vector( Aedes mosquitoes can easily be separated, however, the samples in the field is often incomplete, making it difficult to separate male and female mosquitoes. The goal of this research is to study the effectiveness of the landmark-based geometric morphometric technique to distinguish the sex of male and female Aedes mosquito vectors, including Ae. aegypti, Ae. albopictus, and Ae. scutellaris, in Thailand. Evaluation of wing size by centroid size analysis found that males and females are distinctly different; females are larger than males in three species of Aedes mosquito. The wing centroid size of Ae. aegypti and Ae. albopictus were very similar, however, Ae. scutellaris was smaller than in both other species. The wing shape between sexes was different in all groups of Aedes mosquitoes. The accuracy of the sex's classification of Aedes vectors was quite high (more than $>80 \%$ from the cross-validated reclassification test). The results of this study prove that landmark-based geometric morphometric can distinguish sexes in Aedes vectors which can be used to solve problems in the field when it is necessary to distinguish the sexes of Aedes mosquitoes with damaged samples.
\end{abstract}

Keywords: Landmark-based geometric morphometric analysis, Aedes mosquito, vectors, Thailand

\section{INTRODUCTION}

Aedes mosquitoes (Diptera: Culicidae) are medically important insects and are vectors of yellow fever, dengue fever, chikungunya, West Nile, and the Zika virus, emerging problems worldwide (Service 2008; World Health Organization 2016). In addition, Aedes species were reported in some areas where they can be vectors of Wuchereria bancrofti and Brugia malayi as filarial nematodes (Service 2008). Aedes aegypti (Linneaus) and Ae. albopictus (Skuse) have been considered important vectors of many other arboviruses (Kraemer et al. 2015). Ae. aegypti is a native mosquito in sub-Saharan Africa, while Ae. albopictus is native to southeast Asia (Kraemer et al. 2015). Currently, Ae. aegypti have spread globally to tropical and sub-tropical regions of the world, while Ae. albopictus has spread to islands in the Indian and Pacific Oceans (Leta et al. 2018). The distribution of them increases the risk occurrence of Aedes-borne viral diseases to humans. Diseases such as dengue, Zika, and chikungunya have increased in many areas (Gardner et al. 2017; Vega-Rua et al. 2014).

Thailand is one country affected by mosquito-borne diseases epidemically, especially dengue fever (Chaiphongpachara T 2017; Clark et al. 2005). In 2017, there were 31,843 cases of dengue fever, and its morbidity rate was 48.20 per 100,000 , while the mortality rate was
0.01 per 100,000 (Ministry of Public Health, Thailand 2017). In Thailand, there are two species of Aedes mosquito, including Ae. aegypti and Ae. albopictus, that are medically important and very common. Ae. scutellaris (Walker) is a species of Aedes which was found in sympatry with both Ae. albopictus and Ae. aegypti (Rattanarithikul et al. 2005; Rattanarithikul et al. 2010). While, this species is not reported as a vector, it is still unclear in Thailand how to separate it from Ae. albopictus because of their similar morphology.

At present, research on mosquito-borne diseases is rapidly developing to stop outbreaks, such as the development of vaccines (Schwartz et al. 2015), methods of mosquito control (Chaiphongpachara et al. 2018; Chaiphongpachara et al. 2018), and genetic modification of mosquitoes to reduce the number of vectors in nature (Terenius et al. 2008). Reducing the number of mosquitoes in areas at risk is another way to control mosquito-borne diseases (Benelli et al. 2016). However, the first step in successful mosquito control is to know the species of mosquito vectors in a particular area as the specific behaviour of each species is key to effective control.

Recently, the geometric morphometric (GM) technique has become a popular method for the identification of similar morphological species or species complex of mosquitoes in many countries (Dujardin 2011; Lorenz et al. 2017). It also helps solve the problem of identification of 
mosquitoes collected in the field as the sample is often incomplete, making it difficult to identify (Chaiphongpachara 2018). Advantages of this technique are easy to use, fast, and affordable (Rohlf 2002). For Aedes mosquitoes, the GM technique has been used in Thailand to identify species, including Ae. aegypti, Ae. albopictus, and Ae. scutellaris, and it can separate them from each other (Sumruayphol et al. 2016). Although species identification of Aedes mosquitoes by GM succeeded, using it to distinguish the sexes is still unclear. Typically, male (non-vector) and female (vector) mosquitoes can be easily separated, however in the field, it is difficult to classify the sex if the samples are damaged.

Thus, the goal of this research is to study the effectiveness of the landmark-based GM technique in Thailand to distinguish the sex between male and female Aedes mosquito vectors, including Ae. aegypti, Ae. albopictus, and Ae. scutellaris. It is useful and important to the field of mosquito vector studies to separate mosquitoes by vector and not vector when the Aedes sample is incomplete.

\section{MATERIALS AND METHODS}

\section{Mosquito collections}

Three species of Aedes mosquitoes, including Ae. aegypti, Ae. albopictus, and Ae. scutellaris were collected by dipping immature larvae and pupae stage samples in the area of the Samut Songkhram Province. Samut Songkhram Province was selected as a sample collection area due to the epidemic of dengue fever in this area of Thailand (Chaiphongpachara et al. 2017). Containers in the houses in the Mueang Samut Songkhram District have been surveyed and the larvae were collected in August 2018. The larvae and pupae of the Aedes mosquito samples were reared in the laboratory at the College of Allied Health Sciences, Suan Sunandha Rajabhat University, Samut Songkhram Provincial Education Center, Thailand. Plastic trays containing filtered water were used to rear the mosquito larvae and they were provided dog food once a day. When they developed into adult mosquitoes, they were identified as Aedes species using taxonomic keys based on their morphology (Rattanarithikul et al. 2005; Rattanarithikul et al. 2010).

\section{Wing preparation}

Sixty individual per species of Aedes mosquitoes (30 male and 30 female) were used for the GM analysis. Only right wings of the Aedes mosquito samples were dissected and mounted on glass microscope slides with a 0.08-0.12 mm glass coverslip using Hoyer's mounting medium. After that, Aedes wing samples were photographed using a digital camera connected to a Nikon SMZ745T stereomicroscope (Nikon Corp., Tokyo, Japan).

\section{Landmark-based GM approach}

In this study, CLIC (Collection of Landmarks for Identification and Characterization) software, which is freely available at https://xyom-clic.eu was used for landmark-based GM analyses. Seventeen landmarks (LM) on the wings of each Aedes individual were selected and digitized, as shown in Figure 1. Before GM analysis, all samples in each species of Aedes mosquitoes were digitized twice to compare with the first digitized images for measurement error, which was estimated by a repeatability index (Arnqvist and Mårtensson 1998). For the analysis of GM, raw coordinates of wing samples in each group were superimposed using Generalized Procrustes Analysis (GPA)(Rohlf 1990). This procedure separates size (centroid size) and shape (partial warps $[\mathrm{PW}])$ variables.

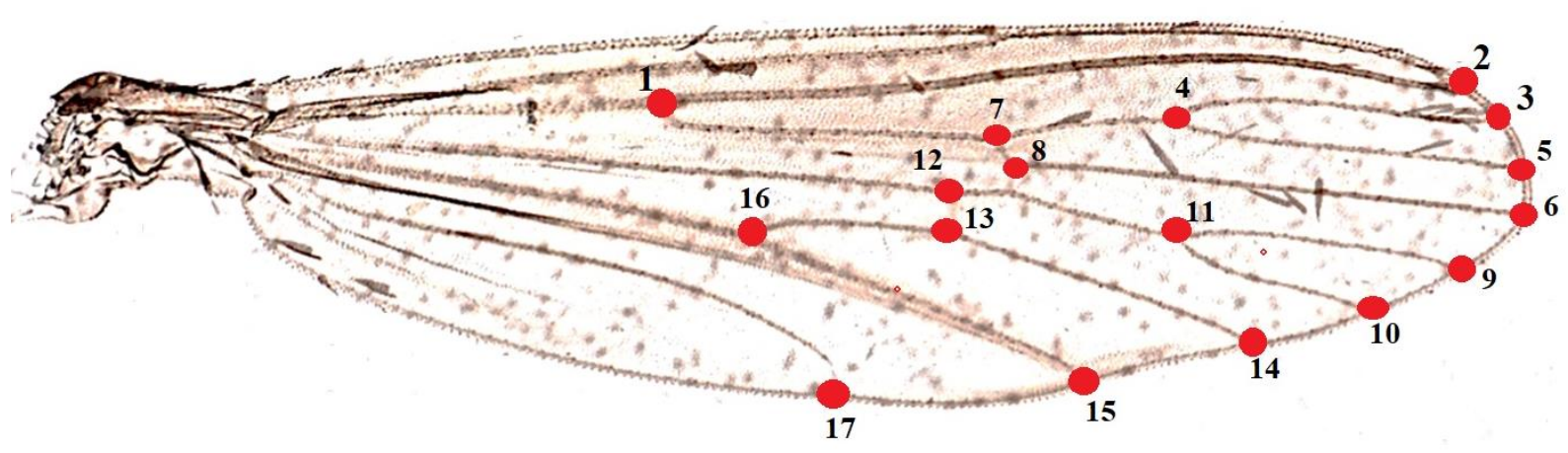

$1 \mathrm{~mm}$

Figure 1. Seventeen LMs on the wing of an Aedes mosquito for GM analyses 


\section{Size variables}

The wing size estimation in male and female Aedes mosquitoes used centroid size (CS), which is defined as the square root of the sum of the squared distances from the centroid to the individual in each landmark (Dujardin 2011). Quantile boxes separating the 25th and 75th quartiles median scores were shown in each box and were created to visualize the CS variations in the males and females of each Aedes species. The statistical significance of average CS difference between males and females in each species was assessed using a non-parametric permutation test (1,000 cycles). A Bonferroni correction was corrected, indicating a statistical significance at $p$ value $<0.05$.

\section{Shape variables}

After GPA, PW as wing shape variables was computed for shape comparison between males and females in each species. The principal components of the PW (or relative warps) were used as input for the discriminant analyses (DA) or canonical variate analysis (CVA), which were used to calculate the factor map for females and males, including Ae. aegypti, Ae. albopictus, and Ae. scutellaris. Then, Mahalanobis distance between sexes was calculated from DA analysis and the difference in each pair was tested using a non-parametric permutation test $(1,000$ cycles) with a Bonferroni correction at $p$-value $<0.05$. Percent accuracy in the separation between males and females used a crossvalidated reclassification test based on the Mahalanobis distance. Finally, a Neighbor Joining tree was created based on Procrustes distances to illustrate morphological divergence between females and males in Aedes species.

\section{RESULTS AND DISCUSSION}

A total of 180 Aedes wings for GM study were divided into 60 wings per species, including 30 male and 30 females for each: Ae. aegypti, Ae. albopictus, and Ae. scutellaris. For this study, we tested the accuracy of seventeen LMs repeatedly marked by a single operator. The results of the measurement error showed that there were good scores for size and shape in the three Aedes species, including 0.98 and 0.96 for size and shape of $A e$. aegypti, 0.95 and 0.96 for size and shape of Ae. albopictus, and 0.93 and 0.97 for size and shape of Ae. scutellaris.

\section{Wing size}

There were wing CS variations between the sexes of the three Aedes species, as shown in Figure 2. In comparison of wing CS between males and females, the wings of females were significantly higher than those of males in all species of Aedes ( $p<0.01$; Table 1). The mean wing CS of Ae. aegypti was very similar, with Ae. albopictus in both sexes ( 2.25 vs. 2.29 in the female and 1.84 vs. 1.83 in the male, respectively). Ae. scutellaris was smaller than Ae. aegypti and Ae. albopictus in both sexes (Table 1).
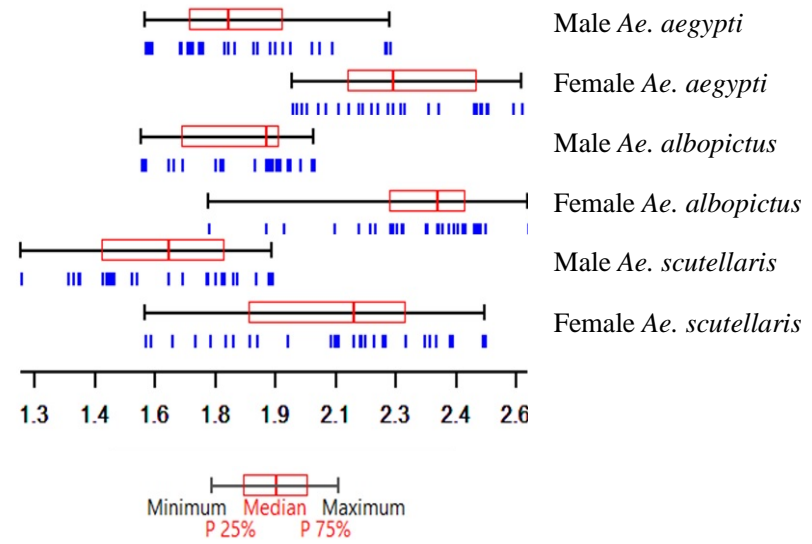

Figure 2. Wing CS variations between sexes (in $\mathrm{mm}$ ), were shown as quantile boxes in which each quantile box shows the median scores separating $25^{\text {th }}$ and $75^{\text {th }}$ quartiles

Table 1. Mean wing CS differences between males and females in each Aedes species

\begin{tabular}{lccc}
\hline Aedes mosquito & $\begin{array}{c}\text { Mean } \pm \text { SD } \\
(\mathbf{m m})\end{array}$ & $\begin{array}{c}\text { Max-Min } \\
(\mathbf{m m})\end{array}$ & $p$-value \\
\hline Male Ae. aegypti & $1.84 \pm 0.04$ & $2.23-1.59$ & $<0.01^{*}$ \\
Female Ae. aegypti & $2.25 \pm 0.03$ & $2.58-1.97$ & \\
Male Ae. albopictus & $1.83 \pm 0.02$ & $2.03-1.58$ & $<0.01^{*}$ \\
Female Ae. albopictus & $2.29 \pm 0.03$ & $2.59-1.75$ & \\
Male Ae. scutellaris & $1.62 \pm 0.04$ & $1.91-1.26$ & $<0.01^{*}$ \\
Female Ae. scutellaris & $2.08 \pm 0.07$ & $2.48-1.58$ & \\
\hline
\end{tabular}

Note: $*=$ significant differences at $p<0.05$

\section{Wing shape}

The mean residual coordinates of both males and females in each Aedes species were superimposed onto the consensus residual coordinates, displaying patterns of shape variation for comparing differences of wing venation between the sexes, as shown in Figure 3. Morphospaces based on wing shape variables from a principal component and factor map of the discriminant factor indicate the differences clearly. The morphospaces found little overlap in male and female groups in the three species (Figures 4A, $\mathrm{B}$, and $\mathrm{C}$ ). While in the factor map, an overlap between the two groups was not found. The highest Mahalanobis distance score (Table 2) is 9.95 of male and female Ae. scutellaris, followed by Ae. albopictus (8.16), and Ae. aegypti (5.43) (The higher the value, the greater the difference of wing shape).

A non-parametric permutation test with a Bonferroni correction test showed statistically significant differences between the sexes in each species $(p<0.01)$. Percent accuracy for sex separation by a cross-validated reclassification test showed quite a high value, providing a mean correct assignment of more than $80 \%$ in all species. Finally, the Neighbor Joining tree based on Procrustes distances of both males and females also confirmed the differences in wing shape between sexes, in which males and females are distinctly separated (Figure 5). 


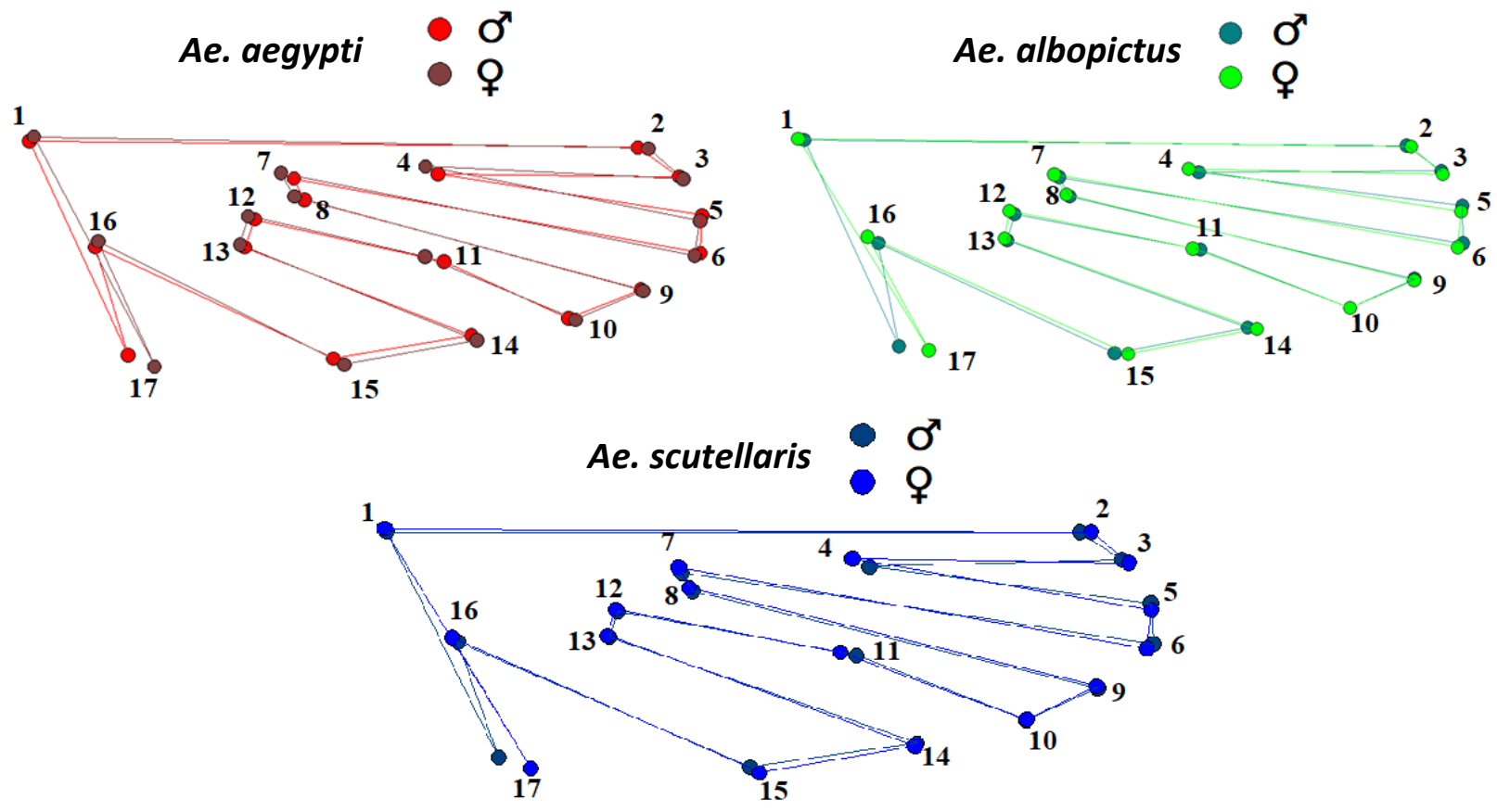

Figure 3. Shape variations between sexes after Procrustes superimposition of 17 landmarks which mean of lines are connecting with landmarks. $($ red $=A e$. aegypti, green $=A e$. albopictus, and blued $=$ Ae. scutellaris $)$

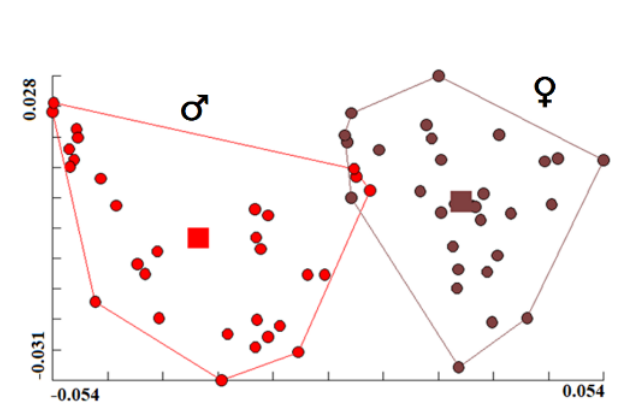

A

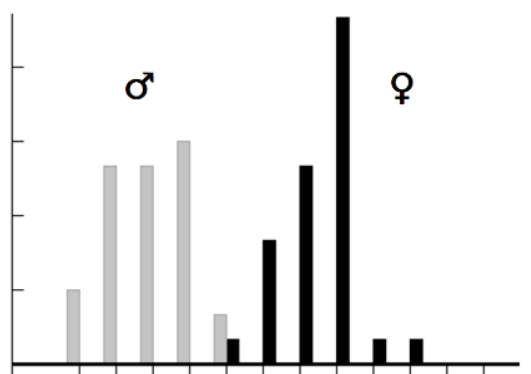

Black = Group $130: 0000015814110$ White = Group 2 30:388920000000 D

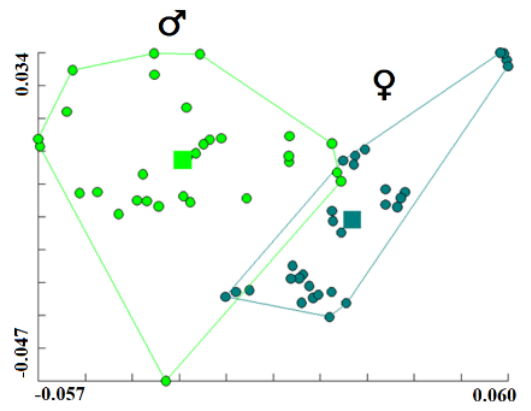

$\mathbf{B}$

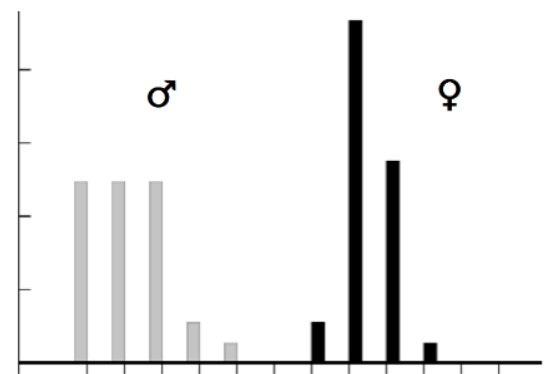

Black = Group $129: 0000000217101$ White = Group 2 30:99921000000 $\mathbf{E}$
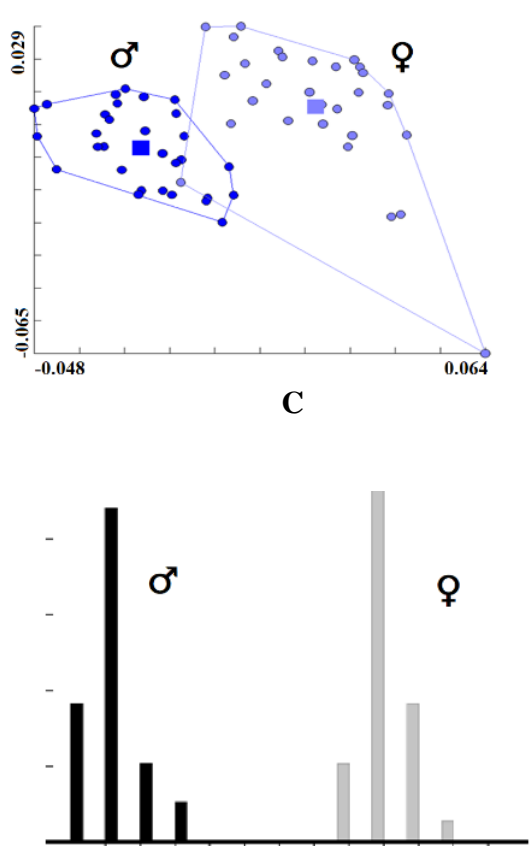

Black $=$ Group $130: 7174200000000$ White $=$ Group 2 30:0000000418710 F

Figure 4. Morphospaces of both male and female mosquitoes based on wing shape variables from principal component, as shown A. Ae. aegypti., B. Ae. albopictus., and C Ae. scutellaris.. While, D. Ae. aegypti., E. Ae. albopictus., and F. Ae. scutellaris were factor maps of the discriminant factor separating males (grey bars) and females (black bars) 


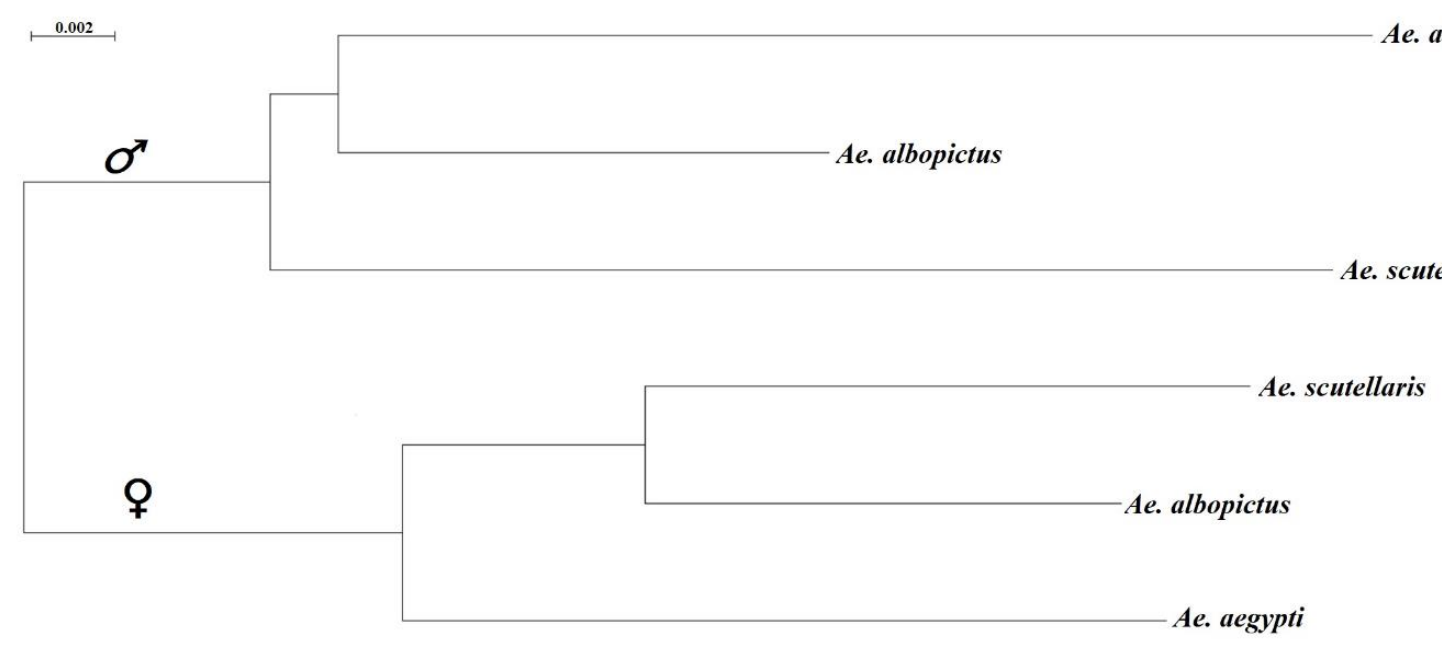

Figure 5. Neighbor joining tree based on Procrustes distances of males and females of three Aedes species

Table 2. Mahalanobis distances between sexes and crossvalidated reclassification scores based on wing shape of Aedes mosquito

\begin{tabular}{lccc}
\hline \multicolumn{1}{c}{ Aedes mosquito } & $\begin{array}{c}\text { Mahalanobis } \\
\text { distances } \\
\text { scores }\end{array}$ & $\begin{array}{c}\boldsymbol{p} \text { - } \\
\text { value }\end{array}$ & $\begin{array}{c}\text { Percent accuracy } \\
\text { in separation } \\
\text { (assigned/observed) }\end{array}$ \\
\hline Male Ae. aegypti & 5.43 & $<0.01$ & $83(25 / 30)$ \\
Female Ae. aegypti & & $<0.01$ & $90(27 / 30)$ \\
Male Ae. albopictus & 8.16 & $100(100 / 30)$ \\
Female Ae. albopictus & & $<0.01$ & $86(26 / 30)$ \\
Male Ae. scutellaris & 9.95 & $93(28 / 30)$ \\
Female Ae. scutellaris & & & $93(28 / 30)$ \\
\hline
\end{tabular}

\section{Discussion}

The sex classification of mosquitoes is initially important for species identification. Typically, in medical entomology, studies focus on mosquito vectors in each area endemic for mosquito-borne diseases (Chaiphongpachara and Sumruayphol 2017). Female mosquitoes are vectors of insect-borne diseases because they must bite humans or animal and feed on the blood to obtain protein and necessary nutrients for the development of their eggs (Killick-Kendrick 1996). Male mosquitoes are not vectors, as they cannot bite but instead feed on nectar and other naturally occurring sugary secretions (Service 1993). The difference between males and females is often observed in the form of the antennae, including the plumose in males, which has long hairs and the pilose in females with a few short hairs (Rattanarithikul et al. 2005). However, if this vital organ is damaged or has disappeared, it may cause a barrier to separate vectors (females) and non-vectors (males). Our results on the landmark-based GM analysis in Aedes wings to distinguish sexes revealed the high efficiency of separation in three Aedes mosquito, including Ae. aegypti, Ae. albopictus, and Ae. scutellaris.

An evaluation of wing size by CS analysis found that males and females are distinctly different; females are larger than males in three species of the Aedes mosquito. The wing CS of Ae. aegypti and Ae. albopictus were very similar, but Ae. scutellaris was smaller than both species. This is in line with previous research that has examined species identification of Ae. aegypti, Ae. albopictus, and Ae. scutellaris in Thailand by GM techniques and found size differences in each species where Ae. scutellaris was the smallest (Sumruayphol et al. 2016). Typically, the identification of mosquito spp. by GM does not use size factors to join in the decision because a variation of size can easily happen from the environment (Sumruayphol et al. 2016; Chaiphongpachara 2018; Wilke et al. 2016). However, for gender discrimination, size factors are important factors that must be used to classify sex.

The wing shape between males and females was different in all groups of Aedes mosquitoes, which corresponds to the results of the wing sizes. Mahalanobis distance value indicates the scores of morphological differences between the sexes in the three Aedes species. Ae. scutellaris had the most differences, followed by Ae. albopictus and Ae. aegypti, respectively. The accuracy of the sex classification of Aedes vectors was quite high (more than $>80 \%$ from a cross-validated reclassification test). In addition, the Neighbor Joining tree showed groupings between sexes, clearly indicating that both males and females were very different in their morphology, which supports the statistical difference by the non-parametric permutation test $(1,000$ cycles $)$ with a Bonferroni correction test $(p<0.01)$.

Wing venation is a unique identity that distinguishes the species of mosquitoes (Wilke et al. 2016). This is consistent with previously research showing that this identity can be used to distinguish mosquito species complex which has a very similar morphology in their group (Sumruayphol et al. 2016). The results of this study prove that landmark-based GM can distinguish sexes in Aedes vectors, which can be used to solve problems of gender discrimination in the field further. 
In conclusions, GM is one of techniques used for solving the problem of species identification and studying variations in populations of mosquito vectors. This study confirms the efficacy of GM for sex classification and supports the application of this technique in the field. In addition, this technique is very interesting and as easy to use, fast, and inexpensive.

\section{ACKNOWLEDGEMENTS}

The authors would like to thank the College of Allied Health Sciences, Suan Sunandha Rajabhat University, Thailand for support and assistance with this project.

\section{REFERENCES}

Arnqvist G, Mårtensson T. 1998. Measurement error in geometric morphometrics: Empirical strategies to assess and reduce its impact on measures of shape. Acta Zool Hung 44; 73-96.

Benelli G, Jeffries CL, Walker T. 2016. Biological control of mosquito vectors: Past, present, and future. Insects. DOI: 10.3390/insects7040052.

Chaiphongpachara T. 2018. Comparison of landmark-and outline-based geometric morphometrics for discriminating mosquito vectors in Ratchaburi Province, Thailand. Biomed Res Intl. DOI: 10.1155/2018/6170502.

Chaiphongpachara T, Bunyuen P, Chansukh KK. 2018. Development of a more effective mosquito trapping box for vector control. Sci World J. DOI: $10.1155 / 2018 / 6241703$.

Chaiphongpachara T, Pimsuka S, Saisanan Na Ayudhaya W, Wassanasompon W. 2017. The application of geographic information system in dengue haemorrhagic fever risk assessment in Samut songkhram Province, Thailand. Int J Geomate 12; 53-60.

Chaiphongpachara T, Sumchung K, Chansukh KK. 2018. Larvicidal and Adult Mosquito Attractant Activity of Auricularia auricula-judae Mushroom Extract on Aedes aegypti (L.) and Culex sitiens Wiedemann. J Appl Pharm Sci 8; 21-25.

Chaiphongpachara T, Sumruayphol S. 2017. Species diversity and distribution of mosquito vectors in coastal habitats of Samut Songkhram province, Thailand. Trop Biomed, 34; 524-532.

Clark DV, Mammen MP, Nisalak A, Puthimethee V, Endy TP. 2005 Economic impact of dengue fever/dengue hemorrhagic fever in Thailand at the family and population levels. Am J Trop Med Hyg. DOI. org/72/6/786 [pii].

Dujardin JP. 2011. Modern Morphometrics of medically important insects. Infect Genet Evol. DOI: 10.1016/B978-0-12-3848901.00016-9.

Gardner L, Chen N, Sarkar S. 2017. Vector status of Aedes species determines geographical risk of autochthonous Zika virus establishment. PLOS Negl Trop Dis DOI: 10.1371/journal.pntd.0005487.

Killick-Kendrick R. 1996. Medical entomology for students. Trans R Soc Trop Med Hyg. DOI: 10.1016/S0035-9203(96)90345-4.

Kraemer MUG, Sinka ME, Duda KA, Mylne AQN, Shearer FM, Barker CM, Hay SI. 2015. The global distribution of the arbovirus vectors Aedes aegypti and Ae. albopictus. ELife. DOI: 10.7554/eLife.08347.

Leta S, Beyene TJ, De Clercq EM, Amenu K, Kraemer MUG, Revie CW. 2018. Global risk mapping for major diseases transmitted by Aedes aegypti and Aedes albopictus. Intl $\mathrm{J}$ Infect Dis. DOI: 10.1016/j.ijid.2017.11.026.

Lorenz C, Almeida F, Almeida-Lopes F, Louise C, Pereira SN, Petersen V, Suesdek L. 2017. Geometric morphometrics in mosquitoes: What has been measured. Genet Evol 54: 205-215.

Ministry of Public Health, Thailand. 2017. Annual report. Bureau of Vector Borne Disease. www.thaivbd.org/n/home.com.

Rattanarithikul R, Harbach RE, Harrison BA, Panthusiri P, Coleman RE, Richardson JH. 2010. Illustrated keys to the mosquitoes of Thailand. VI. Tribe Aedini. Southeast Asian J Trop Med. DOI: 10.1186/17563305-3-5.

Rattanarithikul R, Harrison BA, Panthusiri P, Coleman RE. 2005. Illustrated keys to the mosquitoes of Thailand. I. Background; geographic distribution; lists of genera, subgenera, and species; and a key to the genera. Southeast Asian J Trop Med 36; 1-80.

Rohlf F. 1990. Rotational fit (Procrustes) Methods in: Proceedings of the Michigan morphometrics workshop. Misc publ-Mus Zool, Univ. Mich. www. http://hdl.handle.net/2027.42/49535.com

Rohlf FJ. 2002. Geometric morphometrics in systematics. Morphology, Shape and Phylogenetics, 175-193.

Schwartz LM, Halloran ME, Durbin AP, Longini IM. 2015. The dengue vaccine pipeline: Implications for the future of dengue control. Vaccine. DOI: 10.1016/j.vaccine.2015.05.010

Service M. 2008. Medical entomology for students, fourth edition. Medical Entomology for Students, Fourth Edition. DOI: 10.1017/CBO9780511811012.

Service MW. 1993. Mosquito ecology: Field sampling methods, Second edition. Elsevier, New York.

Sumruayphol S, Apiwathnasorn C, Ruangsittichai J, Sriwichai P, Attrapadung S, Samung Y, Dujardin JP. 2016. DNA barcoding and wing morphometrics to distinguish three Aedes vectors in Thailand. Act Trop 159:1-10.

Terenius O, Marinotti O, Sieglaff D, James AA. 2008. Molecular genetic manipulation of vector mosquitoes. Cell Host Microbe. DOI: 10.1016/j.chom.2008.09.002

Vega-Rua A, Zouache K, Girod R, Failloux AB, Lourenco-de-Oliveira R. 2014. High Level of Vector Competence of Aedes aegypti and Aedes albopictus from ten american countries as a crucial factor in the spread of chikungunya Virus. J Virol. DOI: 10.1128/JVI.00370-14.

Wilke ABB, De Oliveira Christe R, Multini LC, Vidal PO, Wilk-Da-silva R, De Carvalho GC, Marrelli MT. 2016. Morphometric wing characters as a tool for mosquito identification. PLoS ONE 11 (8): e0161643. DOI: 10.1371/journal.pone.0161643.

World Health Organization. 2016. Mosquito Born Diseases. WHO, Geneva. 